Editor's Note: These short reviews of a recent paper in the Journal, written exclusively by graduate students or postdoctoral fellows, are intended to mimic the journal clubs that exist in your own departments or institutions. For more information on the format and purpose of the Journal Club, please see http://www.jneurosci.org/misc/ifa_features.shtml.

\title{
Search for the Neural Correlates of Learning to Discriminate Orientations
}

\author{
Jay Hegdé \\ Department of Psychology, University of Minnesota, Minneapolis, Minnesota 55455 \\ Review of Raiguel et al. (http://www.jneurosci.org/cgi/content/full/26/24/6589)
}

Perceptual learning, or improvement in perceptual abilities with experience or training, is crucial to the ability of an organism to dynamically adapt to its environment. Understanding perceptual learning is important not just because it represents an interesting type of adult plasticity, but also because it is closely related to perception itself. But the neuronal mechanisms of perceptual learning are unclear. A recent single-unit study in the macaque by Raiguel et al. (2006) in the Journal of Neuroscience sheds new light on the neural changes that underlie a prototypical form of visual perceptual learning, namely learning to discriminate orientations.

With extended training, monkeys and humans alike can learn to detect progressively smaller differences in the orientation of a visual stimulus, such as gratings. In other words, the just-noticeable difference (JND) in the orientation of two gratings steadily decreases with training and eventually bottoms out (Yang and Maunsell, 2004; Raiguel et al., 2006). Interestingly, improvements in discrimination at one orientation or visual field location tend to be specific to that orientation or location, and do not transfer well to other orientations or locations. The behavioral parameters of learning, including the JND

Received July 17, 2006; revised July 26, 2006; accepted July 26, 2006.

Correspondence should be addressed to Jay Hegdé, Department of Psychology, University of Minnesota, 75 Riverside Parkway, Minneapolis, MN 55455. E-mail: hegde@umn.edu.

D0I:10.1523/JNEUROSCI.3012-06.2006

Copyright $\odot$ 2006 Society for Neuroscience $\quad$ 0270-6474/06/268877-02\$15.00/0 and the specificity of trained orientations and locations, can vary considerably from one subject to the next (Ghose et al., 2002). Neural correlates of this phenomenon should, at a minimum, be able to explain these key aspects of the learning process (Ghose, 2004).

Early visual cortical areas V1 and V2, which are retinotopically organized and where orientation selective neurons are common, would seem ideally suited to support location- and orientationspecific learning. However, in the macaque monkey, where learning effects at the level of individual neurons can be directly investigated, no conclusive changes that could account for learning at the behavioral level have been demonstrated (Ghose et al., 2002, and the references therein). Of course, this does not necessarily mean that these areas play no role in the learning. For instance, the trainingdependent changes might have been so distributed across the population that they were not discernible at the level of single cells studied one at a time. On the other hand, it is also possible that the training-dependent changes occur at later stages of visual processing. An obvious candidate in this regard is area V4, which represents an intermediate stage of visual processing, is also retinotopically organized, and contains orientation selective cells.

Previously, Yang and Maunsell (2004) found clear neuronal effects of training in $\mathrm{V} 4$, in that neurons at the trained location had stronger responses and narrower tun- ing curves. But Raiguel et al. (2006) go one significant step further. They show that training selectively improved the orientation selectivity of a potentially meaningful subset of neurons at the trained location. These cells, which the authors refer to as the "most informative neurons," are those with the peaks of their orientation tuning curves $\sim 25-65^{\circ}$ away from the trained orientation, so that the steepest portions of their tuning curves are likely to be near the trained orientation. Because even small changes in orientation produce relatively large changes in the response in the steepest part of the tuning curves (Fig. 1A) (Ghose et al., 2002), these neurons could, in principle, convey the information most relevant to discriminating small orientation changes in the vicinity of the trained orientation. The slope of the tuning curve of these cells increased relative to that of other cells, thus selectively improving the orientation discriminability of these cells. The untrained V4 locations in the trained animal, which are arguably equivalent to the trained location before training, do not show these changes. Thus, the selective changes at the trained location can be interpreted as trainingdependent changes. Together, these findings suggest that those neurons that were best suited for the discrimination task at hand, and by and large only those neurons, got better at it with training.

Do these neurons represent neural correlates of orientation discrimination learning? The authors are careful not to claim they are, and rightly so. For one 
thing, the authors do not directly correlate the behavior of one or more of these neurons with either the overall trainingdependent improvements in the JND or the discrimination behavior of the animal on a trial-to-trial basis. For another, the tuning curve parameters of the most informative neurons measured by the authors suggest, but do not prove, that these neurons were best suited for the discrimination task at hand. To mention just one of the many plausible contrary scenarios, it is possible that the neurons that had their orientation peaks $25-65^{\circ}$ away from the trained orientation were so tuned after the training that the steepest parts of the tuning curves were not close enough to the trained orientation (Fig. $1 B$ ). Therefore, the fact that the cells with the largest slopes and the largest slope changes were within a certain range of orientation away from the trained orientation (or vice versa) does not by itself prove that these cells were the most informative. The issue here is not that these neurons could not have been the most informative, but that they may not have been.

There are also more general reasons why the results of Raiguel et al. (2006) may not be as definitive as one might be tempted to grant. First, modeling studies have shown that there are scenarios in which the steepening, and the consequent narrowing, of the tuning curves can actually reduce the information conveyed by a neuron (Seriès et al., 2004). Second, the central premise of Raiguel et al. (2006) is that the steepest part of the tuning curve conveys the most information. This is one plausible scenario, but not the only one. There are coding schemes in which portions of the tuning curves other than their steepest parts convey the most information (Butts and Goldman, 2006, and references therein). For instance, a neuron may act as a maximum likelihood estimator of orientation so that, all other things being equal, the peaks of the tuning curves would convey more information than the slopes would. In this case, the most informative neurons would be those with peaks right around the trained orientation. Ultimately, one cannot specify a priori which neurons are most informative for the task at hand without knowing how the
A.
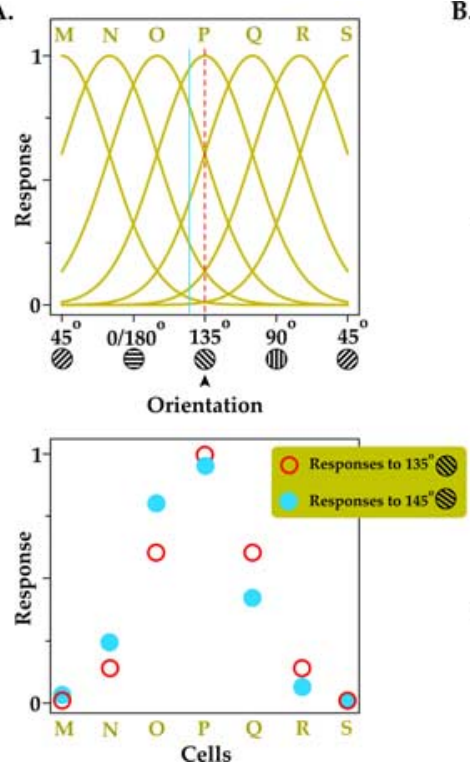
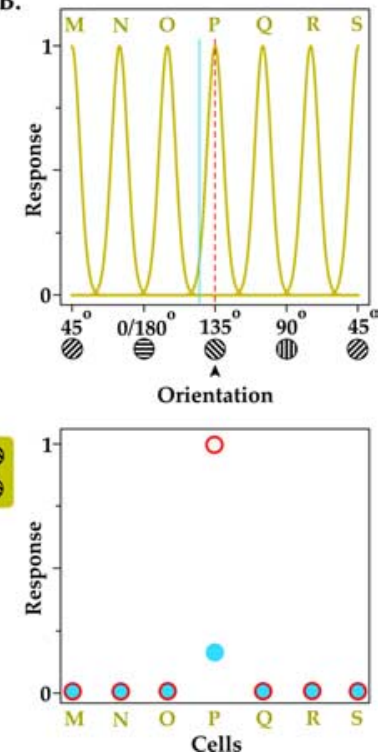

Figure 1. What makes for an informative cell in an orientation discrimination task? A, Top, An idealized network with seven orientation-tuned cells (M-S). The trained orientation of $135^{\circ}$ used by Raiguel et al. (2006) is indicated by the solid arrowhead and the red dashed lines in this panel, and the responses of the seven cells to this orientation are denoted by the red circles in the bottom panel. The test orientation of $145^{\circ}$ to be discriminated from the trained orientation is denoted by the aqua solid line in the top panel, and the corresponding responses are denoted by the aqua dots in the bottom panel. For this discrimination task, the most informative cells are 0 and $Q$, with peaks $\pm 30^{\circ}$ away from the trained orientation, because their responses show the largest difference between the two orientations. The network shown in $\boldsymbol{B}$ (top) is identical to that in $\boldsymbol{A}$, except that the tuning widths are narrower. Note that in this case, the most informative cell is $\mathrm{P}$ (bottom), with its peak coinciding with the trained orientation. Cells 0 and $Q$ are not informative, although their peaks remain at $\pm 30^{\circ}$ away from the trained orientation. Thus, for a given cell to be informative, not only does its peak have to be within an optimal range for the task at hand, but so do many other tuning parameters including, in this case, the tuning widths (or equivalently, slopes).

neural responses are read out and used. Then of course, there is the possibility that neurons elsewhere in the visual system or the brain at large may play a significant role in this form of learning.

It should be noted that these questions are far easier to raise than to answer experimentally. For instance, correlating the response of a given neuron with the discrimination behavior before, during, and after the training would entail chronic recording from the neuron, which is extraordinarily hard to do. To determine whether the steepening of the tuning curves actually helps or hurts in this instance (Seriès et al., 2004), one would need to record simultaneously from a relatively large number of neurons, which is also difficult at best. In view of these technical difficulties, and in spite of the caveats noted above, the case that Raiguel et al. (2006) make for the neuronal changes that may underlie this important type of perceptual learning is rather compelling and intuitively satisfying.

\section{References}

Butts DA, Goldman MS (2006) Tuning curves, neuronal variability, and sensory coding. PLoS Biol 4:e92.

Ghose GM (2004) Learning in mammalian sensory cortex. Curr Opin Neurobiol 14:513-518.

Ghose GM, Yang T, Maunsell JH (2002) Physiological correlates of perceptual learning in monkey V1 and V2. J Neurophysiol 87:1867-1888.

Raiguel S, Vogels R, Mysore SG, Orban GA (2006) Learning to see the difference specifically alters the most informative V4 neurons. J Neurosci 26:6589-6602.

Seriès P, Latham PE, Pouget A (2004) Tuning curve sharpening for orientation selectivity: coding efficiency and impact of correlations. Nat Neurosci 7:1129-1135.

Yang T, Maunsell JH (2004) The effect of perceptual learning on neuronal responses in monkey visual area V4. J Neurosci 27:16171626. 